\title{
ABSOLUTE REGULARITY OF THE NÖRLUND MEAN
}

B. KWEE

(received 15 April 1964)

Let $\left\{p_{n}\right\}$ be any sequence of real or complex numbers subject to the sole restriction

$$
P_{n}=p_{0}+p_{1}+\cdots+p_{n} \neq 0 \quad(n=0,1,2, \cdots) .
$$

And let

$$
t_{n}=\frac{p_{n} s_{0}+p_{n-1} s_{1}+\cdots+p_{0} s_{n}}{P_{n}}
$$

If $t_{n} \rightarrow s$ as $n \rightarrow \infty$, we say that the sequence $\left\{s_{n}\right\}$ is summable Nörlund or summable $(N, p)$ to $s$.

If $t_{n} \rightarrow s$ whenever $s_{n} \rightarrow s$, we say that $(N, p)$ is regular.

It is known [3] that the necessary and sufficient conditions for the regularity of $(N, p)$ are that, for any fixed $k$,

$$
p_{n-k}=o\left(P_{n}\right)
$$

as $n \rightarrow \infty$ and that

$$
\left|p_{0}\right|+\left|p_{1}\right|+\cdots+\left|p_{n}\right|=0\left(P_{n}\right) .
$$

We shall say that the sequence $\left\{s_{n}\right\}$ is absolutely summable Nörlund or summable $|N, p|$ to $s$ if

$$
\sum_{n=0}^{\infty}\left|t_{n}-t_{n-1}\right|<\infty
$$

where $t_{-1}=0$, and

$$
t_{n} \rightarrow s
$$

as $n \rightarrow \infty$.

If (3) and (4) hold whenever $s_{n} \rightarrow s$ and

$$
\sum_{n=0}^{\infty}\left|a_{n}\right|<\infty
$$

where $a_{0}=s_{0}, a_{n}=s_{n}-s_{n-1}(n \geqq 1)$, then we say that $(N, p)$ is absolutely regular.

The aim of this paper is to discuss the relation between regularity and 
absolute regularity of the Nörlund Summability, and for this purpose we require the following theorem.

THEOREM 1. In order that $(N, p)$ should be absolutely regular it is necessary and sufficient that

$$
\sum_{n=m}^{\infty}\left|\frac{P_{n-m}}{P_{n}}-\frac{P_{n-m-1}}{P_{n-1}}\right| \leqq H,
$$

where $H$ is independent of $m$ and $P_{-1}=0$ and that (1) should hold.

For the proof of this theorem we require the following theorem on general summation matrices $\left(c_{n k}\right)$, proved by F. M. Mears [1].

THEOREM. The necessary and sufficient conditions that $\sum_{n=1}^{\infty}\left|u_{n}\right|$, where $u_{n}=U_{n}-U_{n-1}, \quad U_{n}=\sum_{k=1}^{\infty} c_{n k} s_{k}, \quad$ and $s_{k}=a_{0}+a_{1}+\cdots+a_{k}$, should converge whenever $\sum_{n=1}^{\infty}\left|a_{n}\right|$ converges are

(A) $\sum_{k=1}^{\infty} c_{n k}$ converges, for all $n$;

(B) $\sum_{n=1}^{\infty}\left|\sum_{k=m}^{\infty}\left(c_{n k}-c_{n-1, k}\right)\right| \leqq H$, for all $m$ where $H$ is a positive constant.

Proof of Theorem $1{ }^{1}$. The conditions are necessary. We suppose $(N, p)$ is absolutely regular and wish to prove (1) and (6) must then hold. Since

$$
\begin{aligned}
t_{n} & =\frac{p_{n} s_{0}+p_{n-1} s_{1}+\cdots+p_{0} s_{n}}{P_{n}} \\
& =\frac{p_{n}}{P_{n}} s_{0}+\frac{p_{n-1}}{P_{n}} s_{1}+\cdots+\frac{p_{0}}{P_{n}} s_{n},
\end{aligned}
$$

we have

1 An alternative proof of this theorem is possible by appeal to a theorem of H. Hahn, Monatshefte für Math. und Phys. 32 (1922), 3-88. This theorem is quoted in Math. Rev. 9 (1948), 579, by R. P. Agnew in a review of a paper on absolute regularity by Z. Schurr. The theorem of Hahn is as follows.

"Necessary and sufficient conditions that $t_{n}=\sum_{n=0}^{\infty} c_{n k} s_{k}$ should converge (as $n \rightarrow \infty$ ) whenever $s_{n}$ converges absolutely are:

(i)

and then

where

$$
\begin{gathered}
c_{n k} \rightarrow d_{k}, \\
\sum_{k=1}^{\infty} c_{n k} \rightarrow d, \\
\left|\sum_{k=m}^{\infty} c_{n k}\right|<k
\end{gathered}
$$
for all $m$ and $n$;

$$
\begin{gathered}
t_{n} \rightarrow d s+\sum_{k=1}^{\infty} a_{k}\left(s_{k}-s\right), \\
s=\lim _{n \rightarrow \infty} s_{n} .
\end{gathered}
$$

This theorem was pointed out to me by the referee. 


$$
\begin{array}{lr}
c_{n, k}=\frac{p_{n-k}}{P_{n}} & (k=0, \cdots, n), \\
c_{n, k}=0 & (k>n) .
\end{array}
$$

Since $(N, p)$ is absolutely regular Mears's theorem tells us that

$$
\sum_{n=1}^{\infty}\left|\sum_{k=m}^{\infty}\left(c_{n, k}-c_{n-1, k}\right)\right|
$$

is bounded. Putting in the $c_{n k}$ appropriate to $(N, p)$ we find that the above sum is equal to

Hence (6) holds.

$$
\sum_{n=m}^{\infty}\left|\frac{P_{n-m}}{P_{n}}-\frac{P_{n-m-1}}{P_{n-1}}\right| .
$$

Take $s_{k}=1, s_{n}=0(n \neq k)$. Then $\sum_{n=0}^{\infty} a_{n}$ converges absolutely to 0 and hence, when $k$ is fixed,

$$
t_{n}=\frac{p_{n-k}}{P_{n}} \rightarrow 0
$$

as $n \rightarrow \infty$. (1) is also necessary.

The conditions are sufficient. There are two things to be proved:

(i) that, if $s_{n} \rightarrow s$ absolutely, then $t_{n} \rightarrow s$,

(ii) that $t_{n}$ converges absolutely, i.e. $\sum_{n=0}^{\infty}\left|t_{n}-t_{n-1}\right|<\infty$.

Since $\sum_{k=0}^{\infty} c_{n k}$ is a terminating series for each $n$, it is convergent, and Mears's condition (A) is fulfilled. And since, by the algebra above,

$$
\sum_{n=m}^{\infty}\left|\frac{P_{n-m}}{P_{n}}-\frac{P_{n-m-1}}{P_{n-1}}\right|=\sum_{n=1}^{\infty}\left|\sum_{k=m}^{\infty}\left(c_{n k}-c_{n-1, k}\right)\right|,
$$

and the left side is bounded by (6), Mears's condition (B) is fulfilled. So by Mears's theorem, $\sum_{n=0}^{\infty}\left|t_{n}-t_{n-1}\right|<\infty$ and (ii) is established.

To prove that (1) and (6) imply (i), suppose first that $\sum_{n=0}^{\infty} a_{n}$ converges absolutely to 0 . Then we can choose $k$ so that

and

$$
\left|a_{k}\right|+\left|a_{k+1}\right|+\cdots+\left|a_{n}\right|<\frac{\varepsilon}{3 H}
$$

Now, by partial summation,

$$
\left|s_{k-1}\right|<\frac{\varepsilon}{3 H} .
$$

$$
\begin{aligned}
t_{n} & =\frac{p_{n} s_{0}+p_{n-1} s_{1}+\cdots+p_{0} s_{n}}{P_{n}} \\
& =\frac{P_{n} a_{0}+P_{n-1} a_{1}+\cdots+P_{0} a_{n}}{P_{n}} \\
& =A_{n}+B_{n},
\end{aligned}
$$


where

$$
\begin{aligned}
& A_{n}=\frac{P_{n} a_{0}+P_{n-1} a_{1}+\cdots+P_{n-k+1} a_{k-1}}{P_{n}}, \\
& B_{n}=\frac{P_{n-k} a_{k}+P_{n-k-1} a_{k+1}+\cdots+P_{0} a_{n}}{P_{n}} .
\end{aligned}
$$

Since, for all $m \leqq n$,

$$
\begin{aligned}
\left|\frac{P_{n-m}}{P_{n}}\right| & =\left|\sum_{r=m}^{n}\left(\frac{P_{r-m}}{P_{r}}-\frac{P_{r-m-1}}{P_{r-1}}\right)\right| \\
& \leqq \sum_{r=m}^{\infty}\left|\frac{P_{r-m}}{P_{r}}-\frac{P_{r-m-1}}{P_{r-1}}\right| \leqq H
\end{aligned}
$$

we have, by (7),

$$
\begin{aligned}
\left|B_{n}\right| & \leqq\left|\frac{P_{n-k}}{P_{n}}\right|\left|a_{k}\right|+\left|\frac{P_{n-k-1}}{P_{n}}\right|\left|a_{k+1}\right|+\cdots+\left|\frac{P_{0}}{P_{n}}\right|\left|a_{n}\right| \\
& \leqq H\left(\left|a_{k}\right|+\left|a_{k+1}\right|+\cdots+\left|a_{n}\right|\right)<\frac{\varepsilon}{\mathbf{3}} .
\end{aligned}
$$

and, by partial summation,

$$
\begin{aligned}
\left|A_{n}\right| & =\left|\frac{p_{n} s_{0}+p_{n-1} s_{1}+\cdots+p_{n-k+2} s_{k-2}+P_{n-k+1} s_{k-1}}{P_{n}}\right| \\
& \leqq\left|\frac{p_{n} s_{0}+p_{n-1} s_{1}+\cdots+p_{n-k+2} s_{k-2}}{P_{n}}\right|+\left|\frac{P_{n-k+1}}{P_{n}}\right|\left|s_{k-1}\right| .
\end{aligned}
$$

By (8) and (9),

$$
\left|\frac{P_{n-k+1}}{P_{n}}\right|\left|s_{k-1}\right|<\frac{\varepsilon}{\mathbf{3}} .
$$

Since $k$ is a constant, it follows from (1) that

$$
\left|\frac{p_{n} s_{0}+p_{n-1} s_{1}+\cdots+p_{n-k+2} s_{k-2}}{P_{n}}\right|<\frac{\varepsilon}{3}
$$

for all sufficiently large $n$. Therefore

$$
\left|A_{n}\right|<\frac{2 \varepsilon}{3},
$$

and hence, for sufficiently large $n$,

$$
\left|t_{n}\right|<\varepsilon
$$

Thus $t_{n} \rightarrow 0$ as $n \rightarrow \infty$.

If $\sum_{n=0}^{\infty} a_{n}$ converges absolutely to $s$, which is not zero, then $\sum_{n=0}^{\infty} a_{n}^{\prime}$, where $a_{0}^{\prime}=a_{0}-s, a_{n}^{\prime}=a_{n}(n \neq 0)$, converges absolutely to 0 so that 


$$
t_{n}^{\prime}=\frac{p_{n} s_{0}^{\prime}+p_{n-1} s_{1}^{\prime}+\cdots+p_{0} s_{n}^{\prime}}{P_{n}} \rightarrow 0 .
$$

As $n \rightarrow \infty$. But, on substituting $s_{n}-s$ for $s_{n}^{\prime}$,

$$
t_{n}^{\prime}=t_{n}-s \text {. }
$$

Hence $t_{n} \rightarrow s$ as $n \rightarrow \infty$.

If we take $p_{2 n}=1, p_{2 n+1}=0$, we see that (1) and (2) are satisfied but (6) is not when $m$ is an odd integer. Hence a Nörlund method can be regular without being absolutely regular. The question naturally arises as to whether it is true that an absolutely regular Nörlund method is necessarily regular. I have not been able to solve this problem. I have, however, obtained the following two theorems.

THEOREM 2. If $(N, p)$ is absolutely regular and $P_{n}$ is bounded, then $(N, p)$ is regular.

Proof. It follows from the absolute regularity of $(N, p)$ that

$$
\sum_{n=m}^{\infty}\left|\frac{P_{n-m}}{P_{n}}-\frac{P_{n-m-1}}{P_{n-1}}\right|
$$

is bounded and this implies that $p_{0} / P_{m}$ is bounded, so that for all $m$

$$
\left|P_{m}\right| \geqq c>0
$$

where $c$ is a constant.

Now the sum (11), which is equal to

$$
\sum_{n=m}^{\infty} \frac{1}{\left|P_{n} P_{n-1}\right|}\left|P_{n-1} P_{n-m}-P_{n-m-1} P_{n}\right| \text {, }
$$

is bounded. Since we are restricting ourselves to the case in which $P_{n}$ is bounded, $\left|P_{n} P_{n-1}\right|$ is also bounded so that the boundedness of (11) implies the boundedness of

$$
\begin{aligned}
& \sum_{n=m}^{\infty}\left|P_{n-1} P_{n-m}-P_{n-m-1} P_{n}\right| \\
& \quad=\sum_{n=m}^{\infty}\left|\left(P_{n}-p_{n}\right) P_{n-m}-\left(P_{n-m}-p_{n-m}\right) P_{n}\right| \\
& \quad=\sum_{n=m}^{\infty}\left|P_{n} p_{n-m}-p_{n} P_{n-m}\right| \\
& \quad=\sum_{n=0}^{\infty}\left|P_{n+m} p_{n}-p_{n+m} P_{n}\right| .
\end{aligned}
$$

Thus we have 


$$
\sum_{n=0}^{\infty}\left|P_{n+m} p_{n}-p_{n+m} P_{n}\right| \leqq k .
$$

Hence, for any fixed $N$ and all $m$,

$$
\sum_{n=0}^{N}\left|P_{n+m} p_{n}-p_{n+m} P_{n}\right| \leqq k,
$$

and hence

$$
\sum_{n=0}^{N}\left|P_{n+m} p_{n}\right| \leqq k+\sum_{n=0}^{N}\left|p_{n+m} P_{n}\right|
$$

Take $N$ as fixed and make $m \rightarrow \infty$, then

$$
\sum_{n=0}^{N}\left|p_{n+m} P_{n}\right| \rightarrow 0,
$$

because, by $(1), p_{n}=o\left(P_{n}\right)=o(1)$.

Since $\left|P_{n+m}\right| \geqq c>0$, by (12), for all $n, m$, it follows from (13) that

$$
\sum_{n=0}^{N}\left|p_{n}\right| \leqq \frac{k}{c} \text {. }
$$

Hence

$$
\sum_{n=0}^{\infty}\left|p_{n}\right|
$$

converges.

Hence, by (12),

$$
\left|p_{0}\right|+\left|p_{1}\right|+\cdots+\left|p_{n}\right|=0(1)=0\left(P_{n}\right)
$$

and $(N, p)$ is regular.

THEOREM 3. If $(N, p)$ is absolutely regular and $P_{n}$ is not bounded, then $\left|P_{n}\right| \rightarrow \infty$.

Proof. If $\left|P_{n}\right|$ does not tend to infinity, we can find a positive number $G$ such that $\left|P_{n}\right|<G$ for arbitrarily large values of $n$. Also by Theorem 1 ,

$$
\sum_{n=m}^{\infty}\left|\frac{P_{n-m}}{P_{n}}-\frac{P_{n-m-1}}{P_{n-1}}\right| \leqq H
$$

for all $m$. Since $P_{n}$ is unbounded, there is $k$ such that $\left|P_{k}\right|>H G$. Then there is $N>k$ such that $\left|P_{N}\right|<G$. Let $m=H-k$, a positive integer. Then

Hence

$$
\left|\frac{P_{N-m}}{P_{N}}\right|>H
$$




$$
\begin{aligned}
\sum_{n=m}^{\infty}\left|\frac{P_{n-m}}{P_{n}}-\frac{P_{n-m-1}}{P_{n-1}}\right| & \geqq \sum_{n=m}^{N}\left|\frac{P_{n-m}}{P_{n}}-\frac{P_{n-m-1}}{P_{n-1}}\right| \\
& \geqq\left|\sum_{n=m}^{N}\left(\frac{P_{n-m}}{P_{n}}-\frac{P_{n-m-1}}{P_{n-1}}\right)\right| \\
& \geqq\left|\frac{P_{N-m}}{P_{N}}\right|>H,
\end{aligned}
$$

which is in contradiction to (6). Therefore $\left|P_{n}\right| \rightarrow \infty$.

Using the above two theorems, we see that only the case in which $\left|P_{n}\right| \rightarrow \infty$ is left to investigate.

It is worth remarking that it is possible for a Nörlund method to be absolutely conservative without being conservative. This will be shown by an example.

We say that a Nörlund method is conservative if $s_{n} \rightarrow s$ implies $t_{n} \rightarrow t$.

If follows from Theorem 1 of Hardy's book [2] that necessary and sufficient conditions for $(N, p)$ to be conservative are that $(2)$ should hold and that for some $\delta_{m}$

$$
P_{n-m}=\left[\delta_{m}+o(1)\right] P_{n}
$$

as $n \rightarrow \infty$.

A Nörland method is said to be absolutely conservative if (3) holds and $t_{n} \rightarrow t$ whenever $s_{n} \rightarrow s$ and (5) holds.

By Mears's Theorem, a necessary and sufficient condition that $(N, p)$ should be absolutely conservative is that (6) should hold.

We note that (6) does not imply (2). For if we take $P_{n}=e^{n i \theta}$ where $\theta$ is any constant not a multiple of $2 \pi$, we see that (6) is satisfied but (10) is not. Thus the remark is proved.

Finally I should like to express my thanks to the referee for some useful suggestions and to Dr. B. Kuttner for help in writing this paper.

\section{References}

[1] F. M. Mears, Absolute Regularity and the Nörland Mean, Annals of Mathematics 38 (1937), 594-601.

[2] G. H. Hardy, Divergent Series.

[3] C. N. Moore, Summable series and convergent factors.

The University of Malaya,

Kuala Lumpur,

Malaysia. 